\title{
A FWO FLUID MODEL FOR NUCLEAR ROTATIONS
}

\author{
M. A. Z. HABEEB \\ Department of Theoretical Physics, The University of Manchester, Manchester, M13 9PL \\ R. F. BISHOP and J. M. IRVINE \\ Department of Theoretical Physics, The University of Manchester, Manchester, M13 9PL \\ and \\ Science Research Council, Daresbury Laboratory, Warrington, WA4 $4 A D$ \\ and \\ M. R. STRAYER \\ Science Research Council, Daresbury Laboratory, Warrington, WA4 $4 A D$ \\ Received 12 January 1976 \\ (Revised 30 July 1976)
}

\begin{abstract}
A simple macroscopic model based on two-fluid liquid drop ideas is proposed for studying rotational band structure in heavy deformed doubly even nuclei. The pairing and centrifugal degrees of freedom are taken into account in a harmonic approximation. The model is then applied to study the interplay of Coriolis, centrifugal and pairing forces in some well-known backbenders and nonbackbenders and it is concluded that the observed anomalies at high spins could be the result of such an interplay. Calculations for the actinides ${ }^{232} \mathrm{Th}$ and ${ }^{240} \mathrm{Pu}$ are also presented.
\end{abstract}

\section{Introduction}

With the increasing interest in the experimental study of high-spin states in deformed nuclei, strong deviations from simple rigid rotor behaviour have been revealed ${ }^{1}$ ). One such deviation is the anomaly of the moment of inertia at high spins called "backbending".

Many theoretical models have been suggested for the explanation of these phenomena, some of the most common of which are:

(i) The Coriolis-antipairing (CAP) model based on the original prediction of Mottelson and Valatin ${ }^{2}$ ) that the coherent action of the Coriolis force causes a coherent destruction of pairing. This basic idea has been extended in recent years by the incorporation of various microscopic models ${ }^{3-8}$ ).

(ii) The Stephens-Simon model ${ }^{9}$ ), where the Coriolis force causes the rotationaligned (RAL) coupling scheme to be more favoured energetically at high spins, thus leading to band-crossing and possibly backbending.

(iii) Models that suggest shape phase transitions, asymmetries, etc., as causes of backbending. These have been of limited success ${ }^{10-12}$ ). 
The first two effects represent the most widely accepted mechanisms for the explanation of backbending and it is still an open question which is more responsible in the different regions of the periodic table. Excellent reviews of the subject have been given by Johnson and Szymanski ${ }^{13}$ ), Sorensen ${ }^{14}$ ), Stephens ${ }^{15}$ ) and Faessler ${ }^{16}$ ).

Our aim in the present work is the construction of a phenomenological macroscopic model which incorporates the most important ingredients for studying the interplay of pairing, Coriolis and centrifugal forces, and which thus contains the possibility of a phenomenological description of CAP effects and backbending. However, no attempt will be made to include the RAL degree of freedom. It is hoped that such phenomenological models, whose parameters have some direct physical interpretation, might help to achieve a better understanding of the backbending phenomena, and also serve as a guide for more sophisticated microscopic investigations.

The motivation for the present work stems from the success of macroscopic hydrodynamic and phenomenological models at low spins ${ }^{17,18}$ ) and the recent application of two-fluid hydrodynamics to high-spin states ${ }^{19}$ ). The idea of an intermediate nuclear fluid flow ${ }^{20}$ ) is adopted to replace the irrotational flow assumption of previous hydrodynamic models of nuclei. This is consistent with the experimental observation that ground-state moments of inertia are intermediate between what is expected for a superfluid (irrotational) and a normal (rigid) flow. The model thus defined involves two free parameters, with well-defined physical meaning. However, it is found that the results can be further improved by introducing a third parameter whose freedom to vary accounts for some of the major approximations made in the model.

In sect. 2 the model is formulated and in sect. 3 the results of its application to some deformed doubly even nuclei are presented and discussed. Sect. 4 includes a summary and the main conclusions.

\section{Formulation of the model}

The nucleus is viewed as an axially symmetric liquid drop with semiaxes $a$ and $b$, rotating with angular velocity $\omega$ about an axis perpendicular to its axis of symmetry, as shown in fig. 1. We assume that neutrons and protons move as independent noninteracting fluids.

Since the experimental situation indicates intermediate ground-state moments of inertia we seek a nuclear fluid flow that is also intermediate between the superfluid (irrotational) and normal (rigid) flows. Since it is known that the moment of inertia depends on the strength of the pairing interaction ${ }^{14}$ ) it is also necessary that the nuclear fluid flow depends on the pairing interaction.

It was shown by Cusson ${ }^{20}$ ) that such an intermediate flow exists, which in turn is a special case of a more general incompressible linear flow, which is a mixture of 


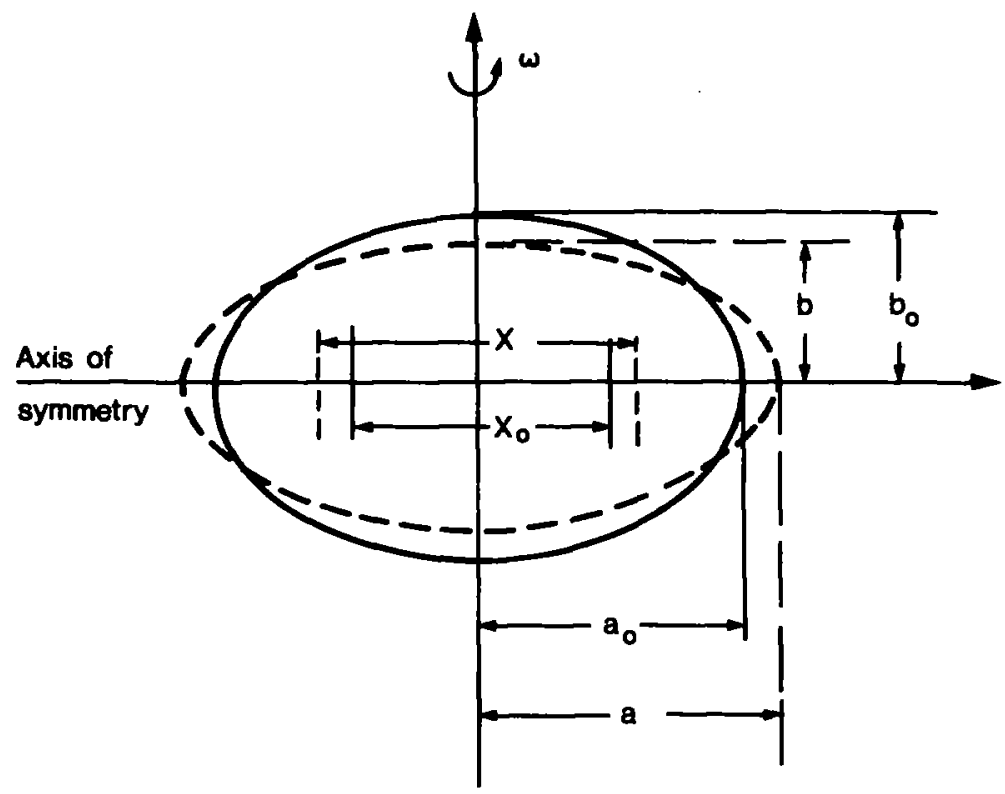

Fig. 1. The rotating nucleus viewed as a spheroid with axial symmetry in equilibrium (solid curve) and stretched (dashed curve) states.

superfluid and normal flows. Cusson ${ }^{20}$ ) also proved that if the ratio of the two velocity-field components, $\gamma$, is fixed before quantization, then the resultant quantized Hamiltonian represents a rotational spectrum with a moment of inertia intermediate between the superfluid and rigid values, depending on the ratio $\gamma$ :

$$
\begin{gathered}
H_{\mathrm{rot}}^{(\tau)}=J(J+1) \hbar^{2} / 2 \boldsymbol{F}_{\mathrm{ri \varepsilon}}^{(\tau)} f_{\mathrm{r}}, \\
f_{\tau}=\left[\gamma_{\tau}+|B|\right]^{2} /\left(1+2 \gamma_{\tau}|B|+\gamma_{\tau}^{2}\right),
\end{gathered}
$$

where $\tau$ labels neutrons $(\tau=\mathbf{n})$ and protons $(\tau=\mathrm{p})$, and $|B|$ is a geometric factor defined by

$$
f_{\text {sup }}^{(\tau)}=B^{2} \cdot f_{\text {rig }}^{(\tau)}
$$

where $\mathscr{S}_{w \mathrm{p}}^{(z)}$ and $\mathscr{S}_{\mathrm{rig}}^{(z)}$ are the pure superfluid and rigid flow moments of inertia respectively and where $J$ is the angular momentum. It can easily be seen that the effective moment of inertia $\mathcal{S}_{\mathrm{r} i}^{(\mathrm{r})} f_{\mathrm{r}}$ in eq. (1) approaches the limits $\boldsymbol{S}_{\mathrm{wp}}^{(\mathrm{z})}$ for $\gamma_{\mathrm{\tau}} \rightarrow 0$ and $\boldsymbol{S}_{\mathrm{rig}}^{(\mathrm{r})}$ for $\gamma_{\mathrm{r}} \rightarrow \infty$.

To produce a pairing-dependent flow, and hence effective moment of inertia, it is necessary to relate the ratio $\gamma_{\tau}$ to the microscopic pairing structure ${ }^{20}$ ). There is no unique way of doing this, but the simplest ansatz that also reproduces the two limits of the moment of inertia $F_{\text {rig }}^{(z)}$ for zero pairing and $\mathscr{S}_{\text {mp }}^{(z)}$ for infinite pairing is ${ }^{20}$ )

$$
\gamma_{\tau} \propto L_{p}^{(\tau)} / R_{0}^{(\tau)}
$$


where $L_{\mathrm{p}}^{(\tau)}$ is the pair correlation length defined by

$$
L_{\mathrm{p}}^{(\tau)}=\frac{2 \varepsilon_{\mathrm{F}}^{(\mathrm{r})}}{k_{\mathrm{F}}^{(\mathrm{r})} v_{\mathrm{z}}}=\text { const } \sqrt{\varepsilon_{\mathrm{F}}^{(\mathrm{r})}} \frac{1}{v_{\tau}},
$$

where $\varepsilon_{\mathrm{F}}^{(\tau)}$ and $k_{\mathrm{F}}^{(\tau)}$ are the Fermi energy and momentum respectively and $v_{\tau}$ is the state-dependent pairing gap parameter ${ }^{21}$ ). Th quantity $R_{0}^{(z)}$ in eq. (4) is a measure of the size of the system, hence, the ratio $\gamma_{\tau}$ as given in eq. (4) represents a qualitative measure of the degree of pairing ${ }^{20,22}$ ). From eqs. (4) and (5) we obtain

$$
\gamma_{\tau}=\xi \sqrt{\varepsilon_{\mathrm{F}}^{(\tau)}} \frac{1}{v_{\tau}},
$$

where $\xi$ is a mass-dependent parameter of the model and it is assumed that neutrons and protons occupy the same region of space, so that we may drop the $\tau$ on $R_{0}$ and $\xi$. To simplify the calculations we assume the density of the fluid $\rho_{\tau}$ to be uniform. It follows from the geometry of fig. 1 and the definitions of rigid and irrotational velocity fields that

$$
\begin{gathered}
\mathscr{f}_{\mathrm{rig}}^{(\tau)}=\frac{4}{15} \pi \rho_{\tau} a b^{2}\left(a^{2}+b^{2}\right), \\
\mathscr{f}_{\text {wp }}^{(\tau)}=\frac{4}{15} \pi \rho_{\tau} a b^{2}\left(a^{2}-b^{2}\right)^{2} /\left(a^{2}+b^{2}\right),
\end{gathered}
$$

and hence from eq. (3)

$$
B^{2}=\left(\frac{a^{2}-b^{2}}{a^{2}+b^{2}}\right)^{2} .
$$

For a system of neutrons and protons the total rotational Hamiltonian is then

$$
H_{\text {rot }}^{(\text {ot })}=J(J+1) \hbar^{2} / 2\left(. F_{\mathrm{rlg}}^{(\mathrm{n})} f_{\mathrm{n}}+. \boldsymbol{S}_{\mathrm{rg}}^{(\mathrm{p})} f_{\mathrm{p}}\right) \text {. }
$$

We make the assumption that centrifugal stretching (CS) is controlled by harmonic restoring forces and thus contributes to the total energy a term of the form ${ }^{18}$ )

$$
V_{\text {cent }}=\frac{1}{2} k\left(X-X_{0}\right)^{2},
$$

where $k$ is a mass-dependent stiffness parameter and $X-X_{0}$ is the displacement from equilibrium of the mass centres of the two halves of the spheroidal nucleus shown in fig. 1.

The important pairing degree of freedom ${ }^{21,23}$ ) is included in the total energy as a harmonic approximation to the pairing energy. This approximation clearly holds rigorously only for low-spin states ${ }^{21}$ ). For high-spin states one expects large changes in $v_{\tau}$ and therefore it is expected that this approximation may break down in the regime in which we are interested. This is clearly one inherent weakness in our model. However, our philosophy is that it is of interest to understand the behaviour of these high-spin states with this simple harmonic form before embarking on more complicated forms that might introduce new adjustable parameters and tend to obscure the basic physics. Also, this harmonic approximation has been used for moderate 
spins by Ma and Tsang ${ }^{24}$ ) and more recently for high spins by Gupta ${ }^{25}$ ). In partial defence of this basic approximation, we note that no appreciable change in our results was found on substituting for the harmonic approximation, the complete form for the BCS pairing energy as used by Ma and Rasmussen ${ }^{21}$ ) in their study of backbending. We conjecture that the fact that the harmonic approximation for pairing is much better in this high-spin regime than one expects beforehand, is in large measure due to the fact that our results indicate at most only a $50 \%$ reduction in $v_{\tau}$ at the backbending rather than a complete collapse of pairing, as indicated later. The harmonic approximation thus achieves a certain degree of internal consistency. For these reasons, we feel justified in using this harmonic approximation as a first step towards an understanding of the model, and to avoid the complications of additional parameters associated with more complicated forms for the pairing energy.

The harmonic form can be derived from number-projected BCS theory in the continuous model (21) as

$$
V_{\mathrm{PBCs}}\left(v_{\tau}\right) \approx \text { const }+\frac{1}{2} C_{v_{\tau}}\left(v_{\tau}-\Delta_{\tau}\right)^{2},
$$

where $C_{v_{\tau}}$ is a pairing-spring constant related to the single particle level density $\rho_{\mathrm{r}}^{l}$ at the Fermi surface, and the ground-state pairing gap $\Delta_{\mathrm{r}}$ and pairing force strength $G_{\tau}$ are as given in ref. ${ }^{21}$ ) where these spring constants are tabulated.

Within our model the total energy $E_{J}\left(v_{n}, v_{p}, X\right)$ is thus the sum of the above contributions for both neutrons and protons

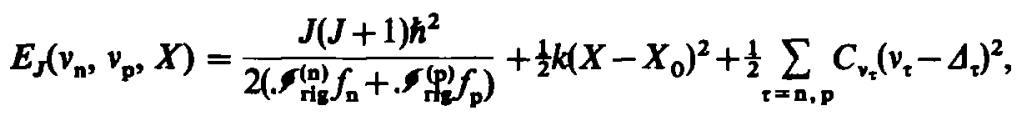

where the constant in $V_{\text {PBCS }}\left(v_{\tau}\right)$ is dropped since we are interested only in calculating excitation energies. To proceed further, we now estimate the ratio $\sqrt{\varepsilon_{\mathrm{F}}^{(\pi)}} / \varepsilon_{\mathrm{F}}^{(\mathrm{p})}$, from the Fermi gas model as (26)

$$
\sqrt{\frac{\varepsilon_{\mathrm{F}}^{(\mathrm{I})}}{\varepsilon_{\mathrm{F}}^{(\mathbf{p})}}}=\left(\frac{N}{Z}\right)^{t}
$$

and utilize the uniform density assumption to write

$$
\begin{gathered}
\rho_{\tau}=\frac{N_{\tau}}{A} \rho, \\
N_{\tau}= \begin{cases}N & \text { for } \tau=n \\
Z & \text { for } \tau=p,\end{cases}
\end{gathered}
$$

where $\rho$ is the total density; and $A, N$ and $Z$ are the mass, neutron and atomic numbers respectively. Eq. (13) may now be written in terms of the stretch variable ${ }^{18}$ ) $T=b / b_{0}$, with the aid of eqs. (7), (9), (14) and (15) and the incompressibility assump- 
tion, as

$$
\begin{aligned}
& E_{J}\left(v_{\mathrm{n}}, v_{\mathrm{p}}, T\right)=\frac{J(J+1) \hbar^{2}}{2.5_{0}} \frac{T^{4}}{1+\eta^{2} T^{6} N F_{\mathrm{n}} A_{\mathrm{p}}+Z F_{\mathrm{p}} A_{\mathrm{n}}} \\
&+\frac{9}{128} k a_{0}^{2} \frac{\left[1-T^{2}\right]^{2}}{T^{4}}+\frac{1}{2} \sum_{\tau=\mathrm{n}, \mathrm{p}} C_{v_{\tau}}\left(v_{\tau}-\Delta_{\tau}\right)^{2},
\end{aligned}
$$

where

$$
\begin{gathered}
\eta=b_{0} / a_{0}, \\
F_{\tau}=\left[\xi \delta_{\tau}\left(1+\eta^{2} T^{6}\right)+\left(1-\eta^{2} T^{6}\right) v_{\tau}\right]^{2}, \\
A_{\tau}=\left[\left(1+\eta^{2} T^{6}\right)^{2} v_{\tau}^{2}+2 \xi \delta_{\tau} v_{\tau}\left(1-\eta^{4} T^{12}\right)+\xi^{2} \delta_{\tau}^{2}\left(1+\eta^{2} T^{6}\right)^{2}\right] \\
\delta_{\tau}= \begin{cases}(N / Z)^{\frac{t}{3}} & \text { for } \tau=n \\
1 & \text { for } \tau=p,\end{cases} \\
\delta_{0}=\frac{4 \pi}{15 A} \rho a_{0}^{5} \eta^{2},
\end{gathered}
$$

and where some constants have been absorbed in $\xi$.

In the application of the model $a_{0}$ and $b_{0}$ are determined from the experimental quadrupole moments $Q_{0}\left[\right.$ ref. $\left.{ }^{27}\right)$ ], assuming a nuclear radius $R_{0}=r_{0} A^{\dagger}=1.2 A^{\dagger} \mathrm{fm}$ and equivalent mass and charge distributions. The gaps $\Delta_{\mathrm{n}}$ and $\Delta_{\mathrm{p}}$ and the pairingspring constants $C_{v_{\mathrm{n}}}$ and $C_{v_{\mathrm{p}}}$ for some of the nuclei studied are taken from $\mathrm{Ma}$ and Rasmussen ${ }^{21}$ ). The total density $\rho$ is assumed to be $A / \frac{4}{3} \pi R_{0}^{3}=0.14$ nucleons $/ \mathrm{fm}^{3}$.

It is found that the results are sensitive to some theoretical and experimental uncertainties as follows:

(i) Small uncertainties in $Q_{0}$ lead to larger uncertainties in $S_{0}$ because $a_{0}$ appears in $\boldsymbol{S}_{0}$ as $\boldsymbol{a}_{\mathbf{0}}$. Similar uncertainties arise from the assumption of equivalent mass and charge distributions. The experimental uncertainty in $r_{0}$ and the possibility that it is different for neutrons and protons also amount to similar uncertainties in $\mathscr{S}_{0}$ since a fixed value of $r_{0}=1.2 \mathrm{fm}$ is used in our calculations.

(ii) The actual mass distributions in nuclei are known not to be uniform. Therefore, $\rho$ should be regarded as an effective density which could well be different from $0.14 \mathrm{nucleons} / \mathrm{fm}^{3}$.

(iii) The non-uniqueness of the ansatz for relating $\gamma_{\varepsilon}$ to $v_{\tau}$ suggests that other possible forms would lead to different predictions. However, some of the alterations could be partly accounted for by altering $S_{0}$.

It is found that the calculational results could be improved if a factor $P$ is introduced to multiply $S_{0}$ in eq. (16). This factor is introduced to accomodate the uncertainties mentioned earlier and allows us to study the behaviour of the results for a range of values of $P$, which could be tolerated within these uncertainties. The final expression for $E_{J}\left(v_{\mathrm{n}}, v_{\mathrm{p}}, T\right)$ is then that of eq. (16), but with $\boldsymbol{S}_{0}$, multiplied by $P$. The energies for different angular momenta are determined from this expression 
by minimisation for a given $J$ with respect to $T, v_{\mathrm{n}}$ and $v_{\mathrm{p}}$, and at the same time adjusting the parameters $\xi$ and $k$ to get the best fit to the experimental spectrum for a given $P$ The interplay of $H_{\text {rot }}^{(\text {tot) }}$ and $V_{\text {eent }}$ in the minimisation with respect to $T$ constitutes centrifugal stretching while its interplay with $V_{\mathrm{PBCs}}$ in the minimisation with respect to $v_{n}$ and $v_{p}$ constitutes the CAP effect in our model. We note here that eq. (16) and the calculational scheme mentioned above resembles an extended VMI model ${ }^{28}$ ) where the single generalized stretch variable is now extended to three variational parameters $T, v_{\mathrm{n}}$ and $v_{\mathrm{p}}$, thus allowing for some differentiation between neutrons and protons, as well as accounting for the CAP effect explicitly.

\section{Discussion of the results}

The results are displayed in figs. $2-8$ in the customary way by plotting $\left(25 / h^{2}\right)$ versus $(h \omega)^{2}$ where these quantities are derived from the spectra using the formulae that give a straight line for the VMI model ${ }^{14}$ ):

$$
\begin{gathered}
\frac{2 \zeta}{\hbar^{2}}=\frac{4 J-2}{\Delta E_{J,(J-2)}}, \\
(h \omega)^{2}=\frac{\left[\Delta E_{J,(J-2)}\right]^{2}(2 J-1)}{\left(\frac{3}{4}\right)^{3}\left\{[J(J+1)]^{3}-[(J-1)(J-2)]^{3}\right\}^{3}} .
\end{gathered}
$$

For the typical nucleus ${ }^{164} \mathrm{Er}_{68}$ we also display plots of the gap $v_{\mathrm{r}}$ and the deformation parameter $\beta_{\mathrm{J}}$ versus $J$ (see fig. 2). The later plots show the CAP and CS effects, as well as the increase in $v_{p}$ and the Coriolis-anti stretching (CAS) effects at the critical spin, similar to what was found in microscopic models ${ }^{3,5-8}$ ). The experimental spectra for all nuclei are taken from ref. ${ }^{29}$ ) and references therein.

The main conclusions from these results are summarised in table 1 , where the three parameters $\xi, k$ and $P$ as well as an estimation of the quality of the empirical fit are given. We achieve a good fit to all nuclei studied, with the exception of ${ }^{170} \mathrm{Yb},{ }^{154} \mathrm{Gd}$, and ${ }^{186}$ Os. However, the behaviour of the high-spin states in these nuclei cannot be attributed solely to a pairing phase transition ${ }^{15}$ ) and since our model does not allow for the possibility of RAL, this effect cannot be excluded, at least in these nuclei. The results for the actinides ${ }^{232} \mathrm{Th}$ and ${ }^{240} \mathrm{Pu}$ are not sensitive to $P$ and show no sign of CAP backbending even at very high spins. This leads us to believe that if backbending occurs in these nuclei at high spins, it must be due to band-crossing or some other phenomena not contained in our model ${ }^{30}$ ).

It should also be mentioned that all backbending found at high spins in these calculations is due to neutron CAP. However, there are also cases which show backbending due to proton CAP at spins above the neutron CAP (see fig. 3a). 

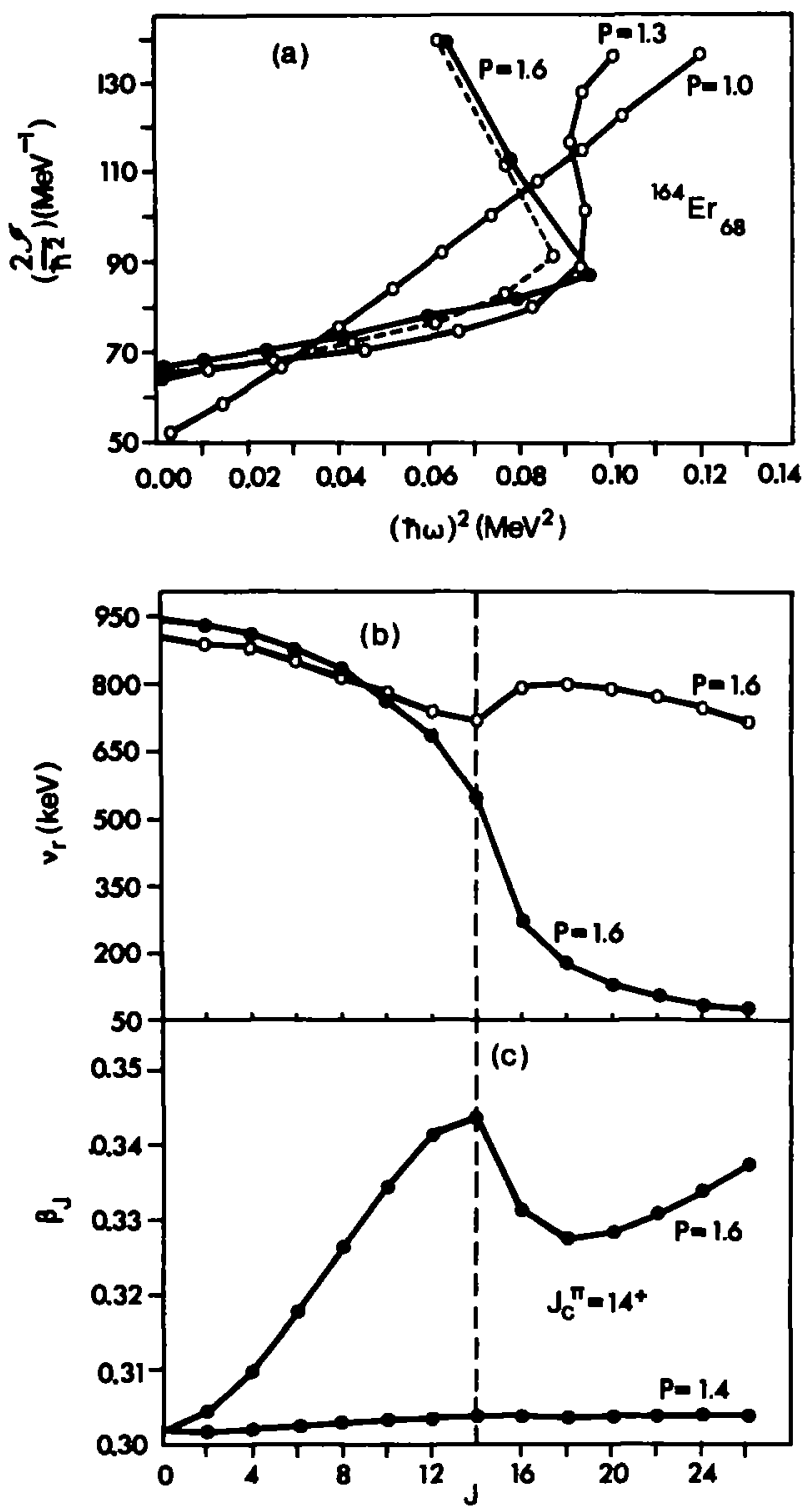

Fig. 2. (a) Experimental (O) and theoretical (O) plots of $\left(25 / h^{2}\right)$ versus $(\hbar \omega)^{2}$ for ${ }^{164} \operatorname{Er}_{68}$. The $P=1.3$ curve is the result of adjusting $\xi$ only since this resulted in the best fit. (b) Neutron $(O)$ and proton $(O)$ pairing gaps $v_{n}$ and $v_{\mathrm{p}}$ respectively as functions of $J$. (c) The deformation parameter $\beta_{J}=\sqrt{\frac{1}{5} \pi(a-b) / R_{0}}$ as a function of $J$. 


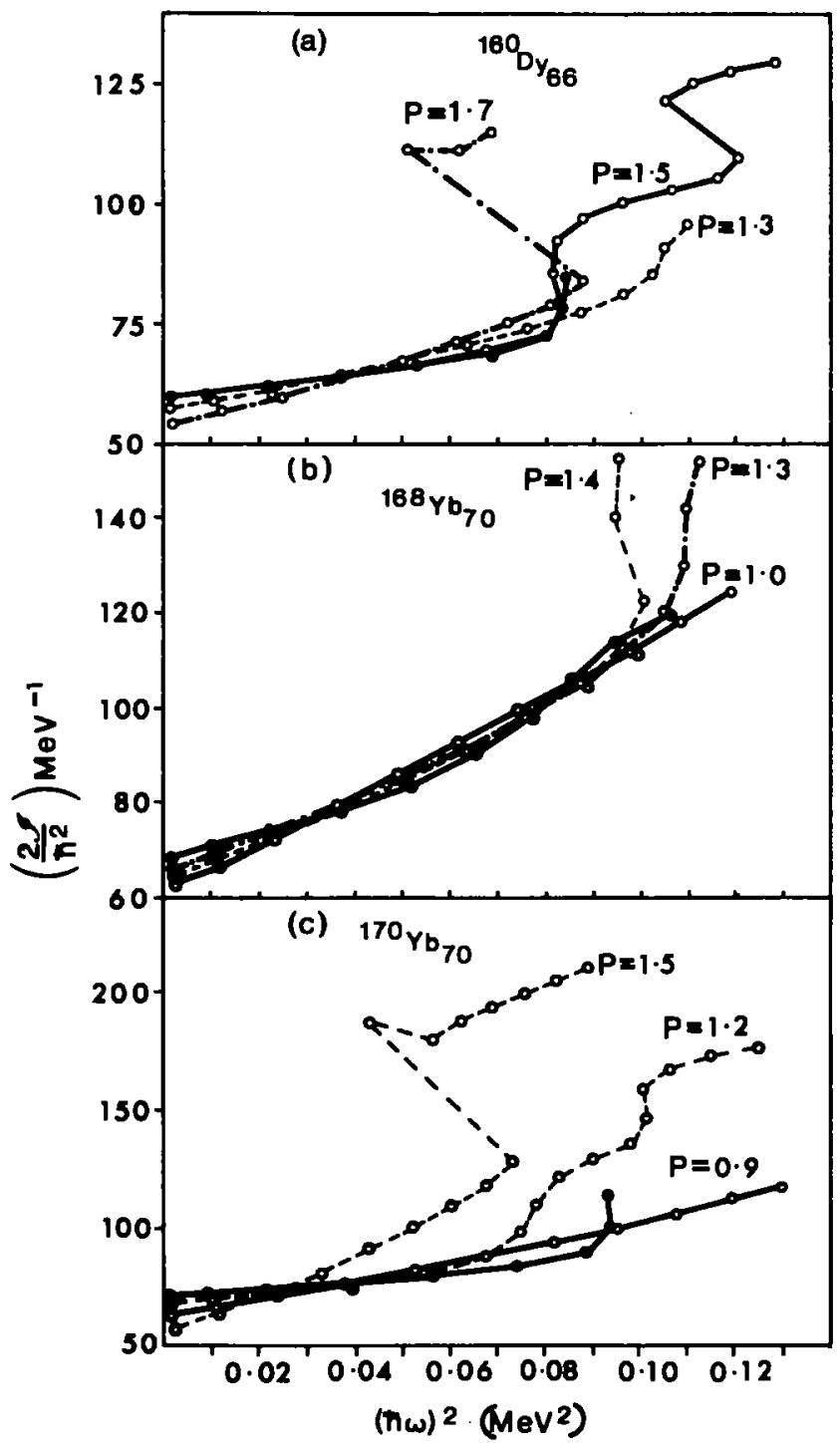

Fig. 3. As in fig. 2a, but for the nuclei (a) ${ }^{160} \mathrm{Dy}_{66}$, (b) ${ }^{168} \mathrm{Yb}_{70}$ and (c) ${ }^{170} \mathrm{Yb}_{70}$. The $P=1.2$ and 1.5 curves for ${ }^{170} \mathrm{Yb}_{70}$ are the results of adjusting $\xi$ only for the same reason as in fig. $2 \mathrm{a}$. 


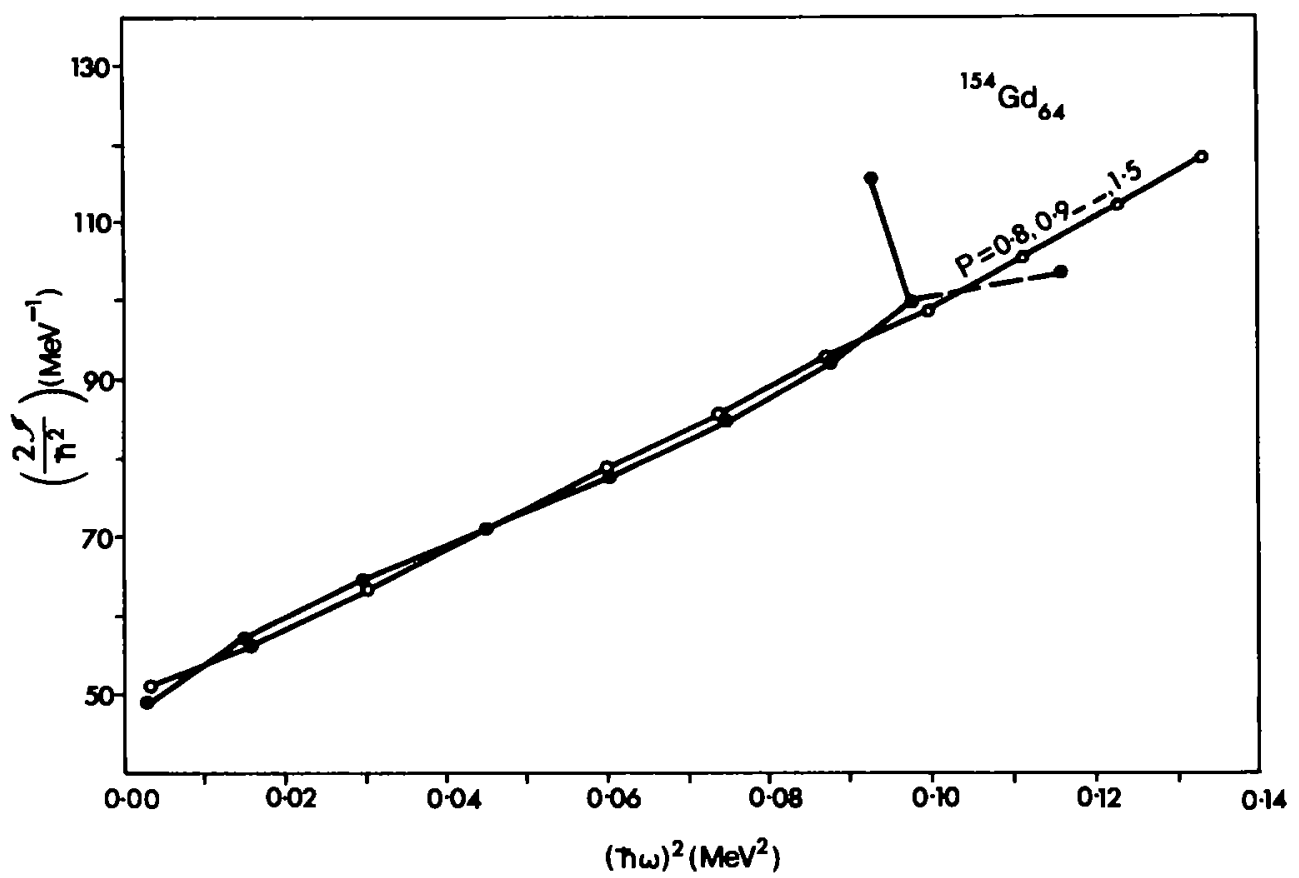

Fig. 4. As in fig. $2 \mathrm{a}$, but for ${ }^{154} \mathrm{Gd}_{64}$. Both experimental forking levels are shown here. Only experimental levels below the forking levels are included in the fit due to the uncertain nature of higher levels.

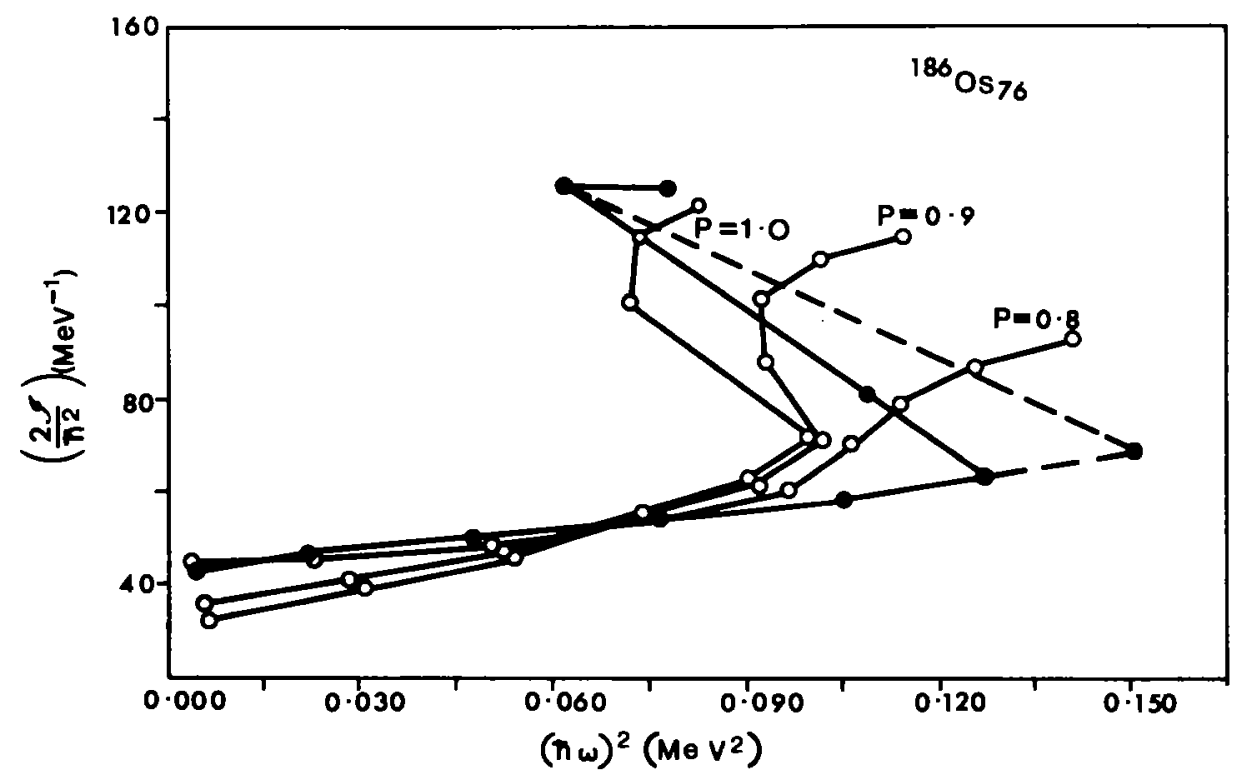

Fig. 5. As in fig. 4, but for ${ }^{186} \mathrm{Os}_{76}$. 


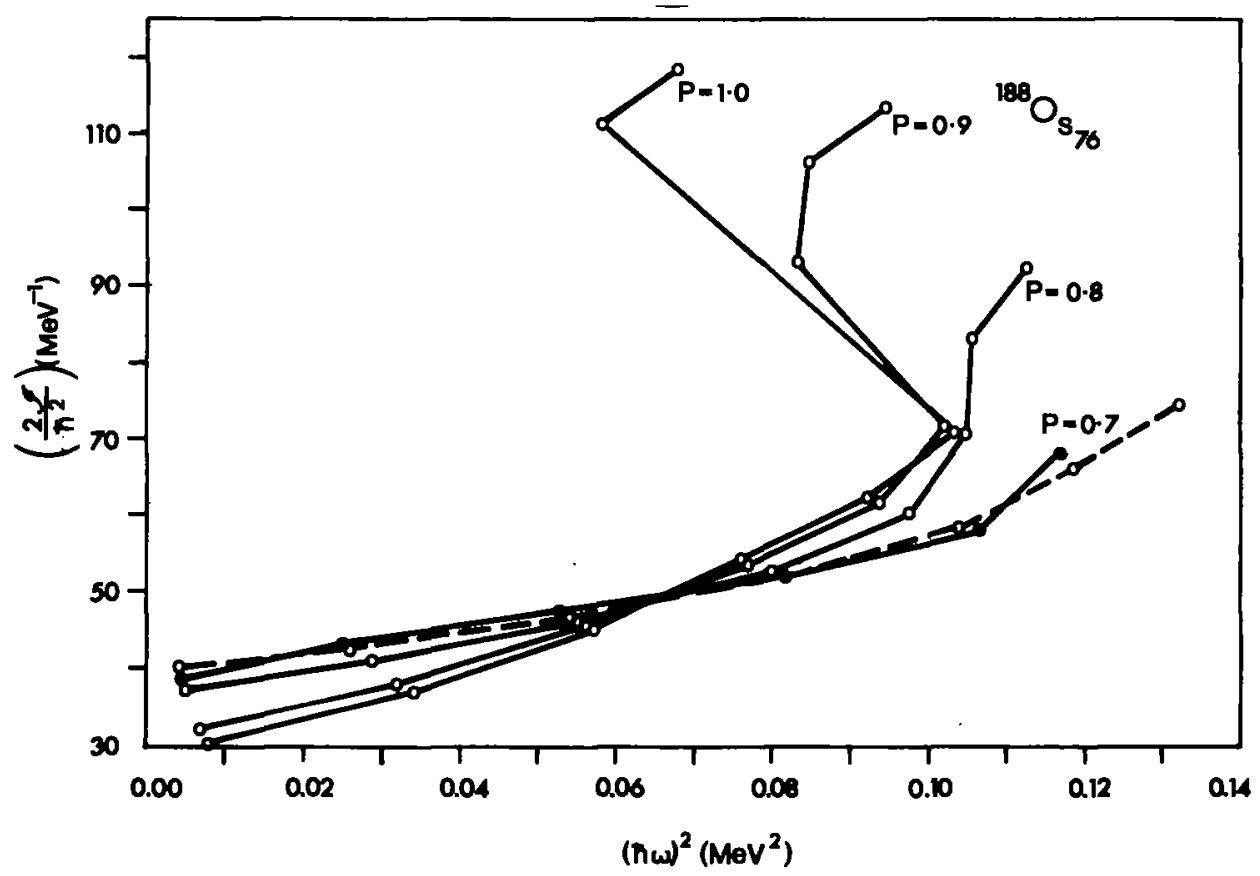

Fig. 6. As in fig. 2a, but for ${ }^{188} \mathrm{Os}_{76}$.

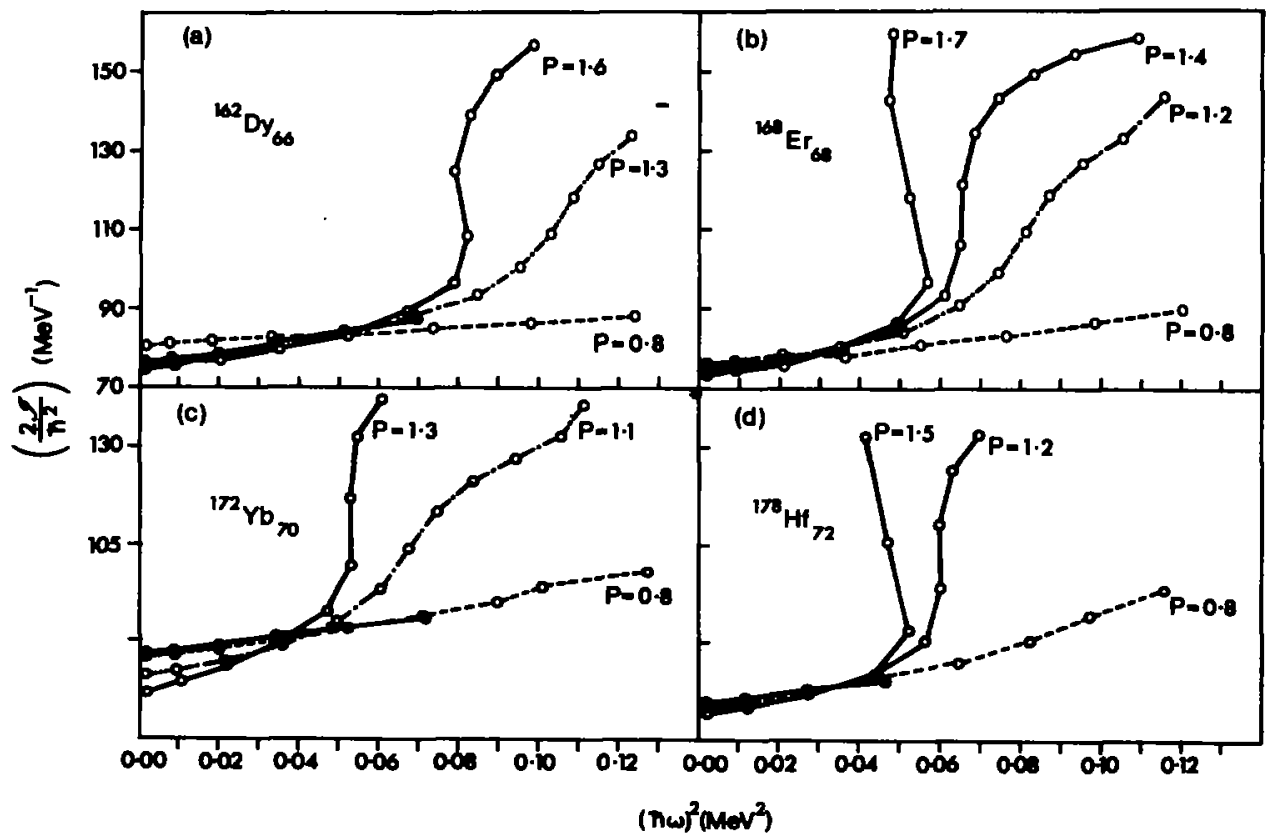

Fig. 7. As in fig. 2a, but for the nuclei (a) ${ }^{162} \mathrm{Dy}_{66}$, (b) ${ }^{168} \mathrm{Er}_{68}$, (c) ${ }^{172} \mathrm{Yb}_{70}$ and (d) ${ }^{178} \mathrm{Hf}_{72}$. All theoretical curves shown, except the $P=1.3$ for ${ }^{172} \mathrm{Yb}_{70}$, are the results of adjusting $\xi$ only for the same reason as in fig. 2 a. 

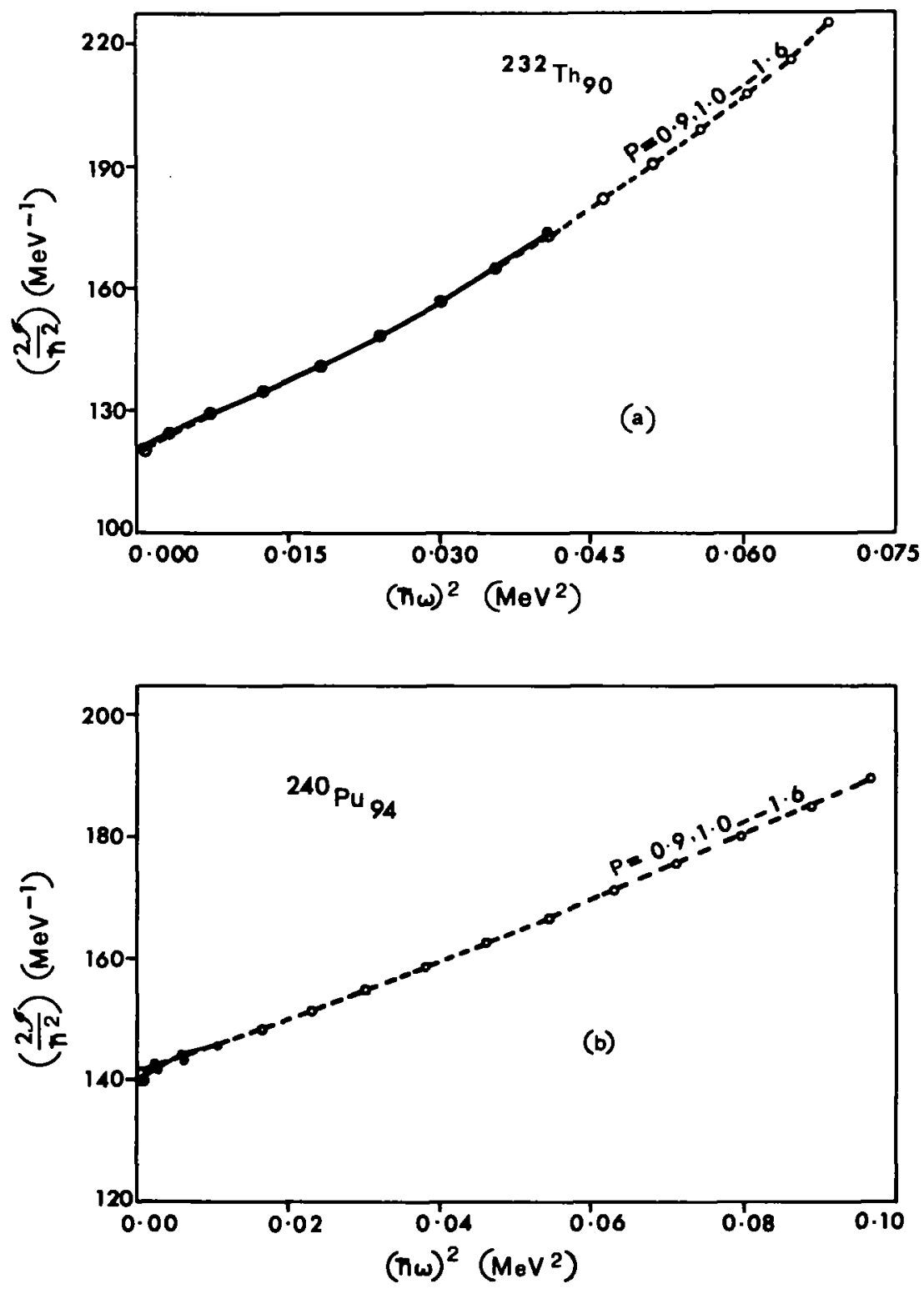

Fig. 8. As in fig. 2a, but for the nuclei (a) ${ }^{232} \mathrm{Th}_{90}$ and (b) ${ }^{240} \mathrm{Pu}_{94}$. Parameters $\Delta_{\mathrm{p}}$ and $\Delta_{\mathrm{p}}$ for these nuclei were calculated from the mass table of ref. ${ }^{32}$ ), then $C_{v_{n}}$ and $C_{v_{p}}$ were calculated as in ref. ${ }^{21}$ ). 
TABLE 1

Table of parameters $k$ and $\xi$ for values of $P$ which give the best $\chi^{2}$ fit to the experimental energies

\begin{tabular}{|c|c|c|c|c|c|}
\hline Nucleus & $P$ & $\begin{array}{c}\xi \\
(\mathbf{k e V})\end{array}$ & $\underset{\left(\mathrm{keV} \cdot \mathrm{fm}^{-2} \times 10^{4}\right)}{k}$ & $\begin{array}{l}\text { Qualitative } \\
\text { fit up to } \\
\text { highest exp. } \\
\text { level }\end{array}$ & Comments \\
\hline${ }^{164} \mathrm{Er}$ & 1.6 & 249.8 & 2.299 & $\begin{array}{c}\text { good } \\
\text { (see fig. 2a) }\end{array}$ & $\begin{array}{l}\text { Backbending is reproduced and } \\
\text { attributed to CAP. No upper } \\
\text { band exp. observed }\end{array}$ \\
\hline${ }^{160} \mathrm{Dy}$ & 1.5 & 323.3 & 1.739 & $\begin{array}{c}\text { good } \\
\text { (see fig. 3a) }\end{array}$ & $\begin{array}{l}\text { Model shows backbending, } \\
\text { whereas exp. curve upbends. No } \\
\text { upper band exp. observed }\end{array}$ \\
\hline${ }^{168} \mathrm{Yb}$ & 1.3 & 325.4 & 0.449 & $\begin{array}{c}\text { good } \\
\text { (see fig. 3b) }\end{array}$ & $\begin{array}{l}\text { Model exhibits upbending at } \\
\text { spins higher than presently mea- } \\
\text { sured. No upper band exp. ob- } \\
\text { served }\end{array}$ \\
\hline${ }^{170} \mathrm{Yb}$ & 0.9 & 439.5 & 0.314 & $\begin{array}{c}\text { bad } \\
\text { (see fig. 3c) }\end{array}$ & $\begin{array}{l}\text { Slight backbending is exp. ob- } \\
\text { served. Model either over-esti- } \\
\text { mates or under-estimates effect. } \\
\text { Other CAP calculations have } \\
\text { similar difficulties "). No upper } \\
\text { band exp. observed }\end{array}$ \\
\hline${ }^{154} \mathrm{Gd}$ & 0.9 & 534.9 & 0.137 & $\begin{array}{c}\text { bad } \\
\text { (see fig. 4) }\end{array}$ & $\begin{array}{l}\text { Band crossing is experimentally } \\
\text { confirmed. Our CAP fits not } \\
\text { sensitive to parameters and show } \\
\text { no backbending. No other CAP } \\
\text { calculations available }\end{array}$ \\
\hline${ }^{186} \mathrm{Os}$ & 0.8 & 384.5 & $\begin{array}{c}\text { large } \\
\text { (no stretching) }\end{array}$ & $\begin{array}{c}\text { bad } \\
\text { (see fig. 5) }\end{array}$ & $\begin{array}{l}\text { Forking is exp. observed and } \\
\text { hence CAP model alone is not } \\
\text { applicable. Model shows no } \\
\text { backbending }\end{array}$ \\
\hline${ }^{188} \mathrm{Os}$ & 0.7 & 393.4 & 1.105 & $\begin{array}{c}\text { good } \\
\text { (see fig. 6) }\end{array}$ & $\begin{array}{l}\text { Fit shows no backbending. No } \\
\text { other CAP calculations avail- } \\
\text { able. No exp. observed upper } \\
\text { band }\end{array}$ \\
\hline${ }^{162} \mathrm{Dy}$ & 1.3 & 432.6 & $\begin{array}{c}\text { large } \\
\text { (no stretching) }\end{array}$ & $\begin{array}{c}\text { good } \\
\text { (see fig. 7a) }\end{array}$ & $\begin{array}{l}\text { Fit does not show CAP back- } \\
\text { bending. Confirms/calculations }{ }^{6} \text { ). } \\
\text { No exp. observed upper band }\end{array}$ \\
\hline${ }^{168} \mathrm{Er}$ & 0.8 & 694.6 & $\begin{array}{c}\text { large } \\
\text { (no stretching) }\end{array}$ & $\begin{array}{c}\text { good } \\
\text { (see fig. } 7 b \text { ) }\end{array}$ & $\begin{array}{l}\text { Fit does not show CAP back- } \\
\text { bending. Confirms calculations"). } \\
\text { No upper band exp. observed }\end{array}$ \\
\hline${ }^{172} \mathrm{Yb}$ & 0.8 & 592.5 & $\begin{array}{c}\text { large } \\
\text { (no stretching) }\end{array}$ & $\begin{array}{c}\text { good } \\
\text { (see fig. } 7 c)\end{array}$ & Same as ${ }^{162} \mathrm{Dy}$ \\
\hline
\end{tabular}


TABLE 1 (continued)

\begin{tabular}{|c|c|c|c|c|c|}
\hline Nucleus & $P$ & $\begin{array}{c}\xi \\
(\mathrm{keV})\end{array}$ & $\left(\mathrm{keV} \cdot \mathrm{fm}^{-2} \times 10^{4}\right)$ & $\begin{array}{l}\text { Qualitative } \\
\text { fit up to } \\
\text { highest exp. } \\
\text { level }\end{array}$ & Comments \\
\hline${ }^{178} \mathrm{Hf}$ & 0.8 & 471.4 & $\begin{array}{c}\text { large } \\
\text { (no stretching) }\end{array}$ & $\begin{array}{c}\text { good } \\
\text { (see fig. 7d) }\end{array}$ & Same as ${ }^{162} \mathrm{Dy}$ \\
\hline${ }^{232} \mathrm{Th}$ & 1.0 & 460.2 & 1.650 & $\begin{array}{c}\text { good } \\
\text { (see fig. 8a) }\end{array}$ & $\begin{array}{l}\text { No CAP backbending, even at } \\
\text { very high spins. Results indepen- } \\
\text { dent of parameter } P\end{array}$ \\
\hline${ }^{240} \mathrm{Pu}$ & 1.0 & 480.7 & 1.896 & $\begin{array}{c}\text { good } \\
\text { (see fig. 8b) }\end{array}$ & Same as ${ }^{232} \mathrm{Th}$ \\
\hline
\end{tabular}

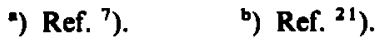

\section{Summary and conclusions}

We have presented a macroscopic hydrodynamical model of CAP backbending which has the following features:

(i) The nucleus is represented as a rotating deformed liquid drop comprised of two fluids which are to be thought of as a normal fluid and a superfluid. The moment of inertia as a funtion of the deformation and the pairing gap is then obtained from geometry and the Cusson ansatz ${ }^{20}$ ).

(ii) There are three free parameters $k, \xi$ and $P$ in the current study. The first two have well defined physical significance. The parameter $P$ is introduced to improve the results and to account for some inherent uncertainties. A more sophisticated model for the nuclear ground state may allow for an elimination of some components of $P$.

(iii) The results demonstrate that a two-fluid model of the kind described in this work shows backbending associated with CAP effects. The model is predictive to the extent that a good fit to the low-spin states prescribes a very narrow range of values for $\boldsymbol{P}$. Within these values of $\boldsymbol{P}$ our predicted high-spin spectrum qualitatively agrees with the experimental results over a wide range of nuclei.

(iv) The model does not allow for the RAL effect, since this is a pure single-particle effect. However, it allows for the CAP effect and we note that at the critical spin $J_{c}$ there is no complete collapse of pairing, but rather a decrease in $v_{\tau}$ of order $50 \%$.

(v) Improvements could be obtained from a more detailed investigation of the ansatz for $\gamma_{t}$, a better treatment of the pairing energy, inclusion of shell effects, etc. Work along these lines is now proceeding ${ }^{31}$ ).

Due to the inherent simplicity of the model, we retain the capability of including additional terms in the Hamiltonian, which will thereby extend the range of phenomena that we may describe. For example, within the spirit of the original 
liquid drop model, extended now to two-fluid hydrodynamics, the only truly macroscopic term that we have omitted is a surface-pairing term. There is also the possibility of extending the model to include vibrations. Clearly, a two-fluid system has an incomparably richer vibrational spectrum than that of a single fluid. For example, in analogy with second sound in liquid ${ }^{4} \mathrm{He}$, a two-fluid system may show a vibrational mode in which the normal and superfluid phases vibrate antiphase with the c.m. remaining at rest. It will clearly be of interest to attempt to extend the present model along these lines.

We should like to acknowledge useful discussions with Prof. M. Mihailović.

\section{References}

1) D. Ward, Int. Conf. on reactions between complex nuclei, Nashville, Tennessee, USA, 1974, vol. 2, ed. R. L. Robinson et al. (North-Holland, Amsterdam, 1974) p. 417

2) B. R. Mottelson and J. C. Valatin, Phys. Rev. Lett. 5 (1960) 511

3) K. Kumar, Phys. Scripta 6 (1972) 270

4) B. Banerjee, H. J. Mang and P. Ring, Nucl. Phys. A215 (1973) 366

5) K. Goeke, J. Garcia and A. Faessler, Phys. Lett. 41B (1972) 557

6) L. Lin and A. Faessler, Phys. Lett. 52B (1974) 261

7) A. Faessler, F. Grümmer, L. Lin and J. Urbano, Phys. Lett. 48B (1974) 87

8) F. Grümmer, K. W. Schmid and A. Faessler, Nucl. Phys. A239 (1975) 289

9) F. S. Stephens and R. S. Simon, Nucl. Phys. A183 (1972) 257

10) B. R. Mottelson, Nuclear structure Symp. of the Thousand Lakes, Jousta, Finland, part 2, 1970, p. 148

11) T. Sugawara-Tanabe and K. Tanabe, Nucl. Phys. A208 (1973) 317

12) R. J. Turner and T. Kishimoto, Nucl. Phys. A217 (1973) 317

13) A. Johnson and Z. Szymanski, Phys. Reports 7 (1973) 181

14) R. A. Sorensen, Rev. Mod. Phys. 45 (1973) 353

15) F. S. Stephens, Proc. Int. Conf. on nuclear physics, Munich, Germany, 1973, vol. I (NorthHolland, Amsterdam, 1973) p. 368; Rev. Mod. Phys. 47 (1975) 43

16) A. Faessler, Int. Conf. on reactions between complex nuclei, Nashville, Tennessee, USA, 1974, vol. 2 (North-Holland, Amsterdam, 1974) p. 437

17 A. Bohr and B. R. Mottelson, Mat. Fys. Medd. Dan. Vid. Selsk. 27 (1953)

18) L. E. Trainor and R. K. Gupta, Can. J. Phys. 49 (1971) 133;

V. R. Prakash, Can. J. Phys. 51 (1973) 1794

19) G. Landi and F. Matera, Nuovo Cim. Lett. 8 (1973) 557

20) R. Y. Cusson, Nucl. Phys. A114 (1968) 289

21) C. W. Ma and J. O. Rasmussen, Phys. Rev. C2 (1970) 798; C9 (1974) 1083

22) A. Bohr and B. R. Mottelson, Nuclear structure, vol. 2 (Benjamin, NY, 1975) p. 398

23) A. N. Mantri and P. C. Sood, Phys. Rev. C7 (1973) 1294

24) C. W. Ma and C. F. Tsang, Phys. Rev. C11 (1975) 213

25) R. K. Gupta, Int. Centre for Theoretical Physics internal report no. IC/75/30, Trieste, March (1975); and Int. Symp. on highly excited States in Nuclei, Sept. 23-26, 1975, Julich, Germany, vol. 1, 1975 , p. 32

26) A. Bohr and B. R. Mottelson, Nuclear structure, vol. 1 (Benjamin, NY, 1969) p. 140

27) K. E. G. Lobner, M. Vetter and V. Hönig, Nucl. Data Tables 7 (1970) 495

28) M. A. J. Mariscotti, G. Scharff-Goldhaber and B. Buck, Phys. Rev. 178 (1969) 1869

29) R. O. Sayer, J. S. Smith III and W. T. Milner, Nucl. Data Tables 15 (1975) 85

30) H. J. Specht, E. Konecny, D. Heunemann and J. Weber, European Conf. on nuclear physics, Aixen-Provence, France, vol. 2, June 26-July 1, 1972, p. 8

31) M. A. Z. Habeeb, R. F. Bishop, J. M. Irvine and M. R. Strayer, to be published

32) V. E. Viola, Jr., J. A. Swant and J. Graber, Atomic Data and Nucl. Data Tables 13 (1974) 35 